This is the pre-peer-reviewed version of the following article: Hlobil, U. (2015), "There Are Diachronic Norms of Rationality." Thought: A Journal of Philosophy. doi: 10.1002/tht3.155, which has been published in Thought.

\title{
There Are Diachronic Norms of Rationality
}

\author{
Ulf Hlobil
}

\begin{abstract}
Some philosophers have recently argued that there are no diachronic norms of epistemic rationality, i.e., that there are no norms regarding how you should change your attitudes over time. I argue that this is wrong on the grounds that there are norms governing reasoning.
\end{abstract}

Some philosophers (e.g. Hedden forthcoming-b, forthcoming-a; Christensen 2000) have recently claimed that there are no diachronic norms of epistemic rationality, where such diachronic norms are norms that "concern how you should change your attitudes over time” (Hedden forthcoming-b, Sec. 2). ${ }^{1}$ It is sometimes assumed that the same idea can be formulated as the claim that what "attitudes you ought to have at a time does not directly depend on what attitudes you have at other times” (Hedden forthcoming-b, Sec. 1). This latter claim is, however, compatible with the claim that there are norms that concern how you should change your attitudes over time. In fact, there are such diachronic norms — or so I will argue.

\footnotetext{
${ }^{1}$ Thomas Kelly (unpublished manuscript) has a critique of such views that is similar to mine. However, his case crucially involves the forgetting of evidence. I think my critique is preferable in this respect, although I agree with Kelly's general view on the issue. Jennifer Carr (2014) also has a discussion and critique of such views; her criticism is very different from mine.
} 
Note that I will not take issue with the claim that what attitudes you ought to have at a time does not depend on your attitudes at other times. My target is the claim that there are no diachronic norms of epistemic rationality.

A lot of the discussion about diachronic norms of epistemic rationality concerns principles like diachronic conditionalization or van Fraassen’s “Reflection.” Authors who think that there are no diachronic norms argue that such principles are incompatible with plausible versions of internalism and that their application requires us to have settled controversial claims about personal identity. I will not talk about such principles. My discussion will focus on reasoning, and I will work with qualitative beliefs and not with credences. As will become clear in due course, I think focusing on credences obscures important issues.

\section{An Argument for Diachronic Norms}

Suppose I am doxastically justified in believing at to that not- $A$, that if $B$, then $A$, and that if not- $B$, then not- $A$. At time $t_{1}$, I have added the belief that not- $B$ to my stock of beliefs, and there is no time between $t_{0}$ and $t_{1}$ at which I am in a third belief state. Now consider two ways in which this might happen. In the first case, I fallaciously infer not- $B$ from not- $A$ and if not- $B$, then not- $A$ by affirming the consequent. In the second case, I competently infer not- $B$ by modus tollens - we are not in a case of transmission failure, etc. In the first case I am less epistemically rational than in the second case. My belief states at $t 0$ and at $t_{1}$ are identical. Hence, I must have violated a norm of diachronic rationality in the first case but not in the second. Therefore, there are diachronic norms of epistemic rationality.

We can formulate this argument as follows: 
(1) The two cases do not differ with respect to the doxastic states of the agent but only with respect to the transitions between these states.

(2) The agent violates an epistemic norm only in the first case.

(3) Therefore, there are epistemic norms that govern transitions between doxastic states.

The norm I violate in the first case does not (or not only) tell me what attitudes to have at one time based on attitudes I have at another time. After all, the two cases do not differ with respect to the attitudes I have at either point in time. Nevertheless, in the first case, I violate a norm that concerns how I should change my attitudes over time. For the norm tells me not to infer by affirming the consequent. Such a norm is neither in conflict with internalism nor does it require us to have answered controversial questions regarding personal identity.

I suspect that the reason why philosophers who claim that there are no diachronic norms of epistemic rationality don't realize that their arguments do not establish this conclusion is that the debate is conducted within a Bayesian framework. This framework builds in deductive closure. Thus, it is difficult to model logical reasoning in this framework. Hence, the diachronic norms that are relevant to the kind of case I have described are invisible from the perspective of Bayesian epistemology.

\section{Potential Objections}

In order to resist my argument, an opponent must either deny (1) or (2), or she must question the relevance of (3). 


\subsection{Objections to (1)}

Let me begin with worries regarding (1), i.e. the claim that the two cases that I have described do not differ in the doxastic states of the agent but only in the transition between the states.

Objection 1: Inference always yields a state of believing the conclusion based on the premises. So the two belief states at $t_{1}$ differ because they embody different basing relations; the basing relations in the first case are less rational than those in the second. This explains the difference in rationality. ${ }^{2}$ I have two things to say in response: First, it is not clear that inferences always establish corresponding basing relations. Whitehead and Russell, e.g., point out that deductions do not always line up with basing relations.

In mathematics, the greatest degree of self-evidence is usually not to be found quite at the beginning, but at some later point; hence the early deductions, until they reach this point, give reasons rather for believing the premises because true consequences follow from them, than for believing the consequences because they follow from the premisses. (Whitehead \& Russell 1963, p. v)

If we can reason in accordance with deductions like those in Principia, the same should hold for inference, or at least for inference under suppositions. If that is correct, no basing relations should be established, e.g., by inferences from self-evident truths to self-evident truths. And we can stipulate that all the propositions I believe at $t_{1}$ are self-evident. So we need not concede the claim on which the objection rests. Second, even if there always needs to be a time when the conclusion of an inference is based on the premises - so that there is a time at which the first and the second case differ with respect to the doxastic states of the subject —, this does not undermine the crucial point. For we can imagine a

\footnotetext{
${ }^{2}$ Although this might be questioned, I want to grant my opponent that she can make sense of basing relations without appealing to diachronic norms. If a causal theory of basing is correct, this might be difficult. I will come back to the issue below.
} 
third case that is exactly like the first with respect to basing relations but where the subject does not acquire the belief that not- $B$ by reasoning — perhaps an evil daemon makes sure that the beliefs and basing relations are identical to those in the subject making the fallacious inference. The subjects in the first and in the third case are both irrational. However, only the subject in the first case has reasoned fallaciously. So the subject in the first case is guilty of committing a fallacy while the subject in the third case is not. All other irrationality is shared between the two subjects. Thus, the subject in the first case is less rational than the subject in the third case. Hence, the subject in the first case must have violated a diachronic norm. So even if we concede the opponent's claim about basing relations, we can modify the argument so as to get around the objection.

Objection 2: In the first case, the subject has an irrational disposition at to, which the subject in the second case lacks. Answer: We can stipulate that the subject in the second case also has the disposition to reason by affirming the consequent but does not manifest this disposition. She is still more rational. The manifestation of the disposition, however, is a diachronic phenomenon. And norms governing the manifestations of such dispositions are, hence, diachronic norms.

Objection 3: Since the transitions in the two cases differ, there must be some point in time where there is a difference between the mental states of the subjects. The subject in the first case is less rational because she violates a norm governing some state that she is in at some time, $t_{x}$, between $t_{0}$ and $t_{1}$. Answer: If the agent had stopped at $t_{x}$, i.e. when she reached the state that violates a synchronic norm, she would not have committed the fallacy of affirming the consequent. Otherwise, the reasoning was already over at $t_{x}$. So according to my opponent's view, the problem with the first case is not that the subject 
reasoned by affirming the consequent. I can concede that there might be a further problem. But whatever norm the subject has violated at $t_{x}$, she has not yet committed the fallacy of affirming the consequent. So the subject that stops at $t_{x}$ is more rational than the subject in the first case that continues until $t_{1}$. My opponent cannot explain this difference in rationality.

Very roughly, something close enough to (1) must be right because the problem in the first case is that the subject reasons fallaciously. Pointing to any synchronic difference won't amount to pointing to the right kind of irrationality. Even if there are synchronic differences, the difference between the transitions brings with it a particular kind of difference in rationality and this difference cannot be captured by talking only about static phenomena.

\subsection{Objections to (2)}

Let me now turn to objections to premise (2). Such objections must either say that the subject in the second case is epistemically irrational or that the subject in the first case is not. The Objection 4 is of the first kind; Objections 5 and 6 are of the second kind.

Objection 4: The belief state of the subject in the first case is not synchronically rational because it is not closed under consequence. Answer: The epistemic badness of the first case does not stem from the failure of deductive closure. Otherwise, it would also be present in the second case.

Objection 5: Correct reasoning is merely instrumentally valuable; it is only insofar as reasoning reliably leads to the fulfilment of synchronic norms that it is good. Considered by themselves, there is nothing wrong with fallacious inferences. I have two things to say 
in response: First, imagine you could have an apparatus implanted in your brain that prevents you from reasoning but makes sure that your way of acquiring beliefs is a maximally reliable way for complying with the synchronic norms, given incoming evidence. I find it implausible that having the implant is just an improvement; you are clearly losing out on something if you have the implant. Whether or not you should, all things considered, go ahead and have the apparatus implanted, it will prevent you from attaining an epistemic good, namely the good of reasoning correctly. Second, you cannot justify rules of deductive inference by appeal to their reliability. After all, even if we lived in a world in which whenever a conditional held its convers did too, reasoning by affirming the consequent would still be fallacious.

Objection 6: The dynamic phenomenon of inferring is not relevant to assessments of rationality; only the states of believing-for-reasons that often result from inferences can be assessed in terms of rationality. If the two cases do not differ with respect to believing-forreasons, there is no difference in rationality. Answer: I cannot see what should be wrong with saying that making fallacious inferences is irrational. Perhaps we could doubt this if we had already established that there are no diachronic norms of rationality, but that is precisely what is at issue.

In general, the claim that someone who reasons fallaciously need not violate any epistemic norms is a prima facie counterintuitive implication of the idea that there are no diachronic epistemic norms. The claim cannot be used in a defense of this idea. Hence, denying premise (2) is not a promising option for my opponent. 


\subsection{Doubts about the Relevance of (3)}

Let me now turn to objections according to which (3) is true but irrelevant.

Objection 7: Diachronic norms are derivative norms; synchronic norms generate norms to the effect that (a) one should have a general disposition to comply with the synchronic norms and (b) one should do what someone who has such a disposition would do (see Hedden forthcoming-a, Sect. 10.1). The second of these norms explains the difference in rationality between the two cases. I have three things to say in response: First, we know from standard objections to virtue ethics that doing what someone with the right dispositions would do is not always the right thing to do for someone who lacks these dispositions. Hence, it is not clear that we can really derive the relevant diachronic norms from a norm governing dispositions. Perhaps the one with the irrational dispositions comes closest to complying with the synchronic norms by reasoning fallaciously. Second, if synchronic rationality requires deductive closure, someone who always complies with the synchronic norms will not need to engage in deductive reasoning at all. So it is not clear that someone who has a general disposition to comply with all synchronic norms will have dispositions to reason deductively in particular ways. Perhaps her dispositions to comply with all synchronic norms are of some other kind. Hence, even if we could derive the norm that you should do what someone with such a disposition would do, it might not have any implications for how you ought to reason deductively. Third, the claim of the opponent has changed from the claim that there are no diachronic norms to the claim that diachronic norms are derivative norms. However, diachronic norms often seem more fundamental than synchronic ones. It seems plausible, e.g., that, at least sometimes, one can become doxastically justified in believing a conditional by reasoning in accordance 
with conditional proof. Thus, the norms governing reasoning in accordance with conditional proof seem to be more fundamental than the norms governing doxastic justification for conditionals.

Objection 8: Norms of epistemic rationality must hold necessarily, but norms governing reasoning depend on contingent features of the psychology of the creatures whose reasoning they govern. So norms of reasoning should not count as norms of epistemic rationality (see Hedden forthcoming-a, Sect. 10.2). Answer: We must distinguish two theses. First, the opponent might hold that creatures with different psychological makeups can be governed by genuinely different norms of reasoning. Second, the opponent might hold that agents with certain cognitive limitations should not be criticized for violating norms of rationality when they could not possibly follow these norms. The second thesis is compatible with the claim that the norms of reasoning hold necessarily. It is just that we exempt some agents from an obligation to obey the norms because of something like an ought-implies-can principle. On this view, there aren't really pieces of reasoning that are good for one agent but bad when they occur in a different kind of agent. We are just harsher in applying the norms of reasoning with respect to some agents than with respect to others. By contrast, if the opponent holds the first thesis, she thinks that there are pieces of reasoning that are good for one kind of agent but bad for another, and not because of some kind of exemption because of cognitive limitations. This is implausible. If a piece of reasoning is good, it is good no matter who engages in it. It is just that for some agents, we cannot expect them to do so and we don’t hold them responsible for not engaging in such a piece of reasoning. 
Objection 9: The view I am attacking is only concerned with the justification of beliefs (see Hedden forthcoming-a, Sect. 10.1). Since, in both cases, I have exactly the same evidence at both points in time, there is no difference in propositional justification. And since the basing relations do not differ, there is not even a difference in doxastic justification. So the truth of (3) does not threaten the view at issue. I have two things to say in response: First, compliance with norms governing inference can matter for doxastic justification, as in reasoning in accordance with conditional proof. So it is not clear that my opponent can give an account of doxastic justification in terms of synchronic norms. (If my opponent's claim is restricted to propositional justification, this will raise questions like whether propositional justification must be explained in terms of doxastic justification or vice versa, etc. But pursuing such questions would lead us too far afield.) Second, this response concedes that there are diachronic norms of rationality, and that is what I care about. If the opponent concedes that there are epistemic norms governing how you should change your attitudes over time, we have reached agreement on the point that is at issue in this paper.

\section{Conclusion}

Whatever the status of principles like diachronic Conditionalization or Reflection might be, there are diachronic norms of epistemic rationality. Such norms need not evaluate a belief state at one time based on a belief state at another time; they may evaluate transitions between such states. 


\section{References}

Carr, J. (2014). Don’t stop believing. Available at <http://web.mit.edu/jrcarr/www/pdfs/dontstopbelieving.pdf>

Christensen, D. (2000). Diachronic coherence versus epistemic impartiality. Philosophical Review 109: 349-371.

Hedden, B. (forthcoming-a). Reasons without persons: rationality, identity, and time. Oxford: Oxford University Press. (forthcoming-b). Time-slice rationality. Mind.

Whitehead, A. N. \& Russell, B. (1963). Principia mathematica (2nd ed.). Cambridge: Cambridge University Press. 